\title{
Airways diseases: insights from the European Respiratory Society Annual Congress 2017
}

\author{
Alexander G. Mathioudakis ${ }^{1,2}$, Imran Satia ${ }^{1,2}$, Ian M. Adcock ${ }^{3}$ \\ ${ }^{1}$ Division of Infection, Immunity and Respiratory Medicine, The University of Manchester, Manchester, UK; ${ }^{2}$ Department of Respiratory Medicine, \\ University Hospital of South Manchester, Manchester, UK; ${ }^{3}$ Airways Disease Section, National Heart and Lung Institute, Imperial College London, \\ London, UK \\ Correspondence to: Alexander G. Mathioudakis, MD, MRCP(UK). Academic Clinical Fellow in Respiratory Medicine, Division of Infection, Immunity \\ and Respiratory Medicine, University Hospital of South Manchester, The University of Manchester, Manchester, UK. Email: a.mathioudakis@nhs.net.
}

Submitted Aug 14, 2017. Accepted for publication Sep 18, 2017.

doi: $10.21037 /$ jtd.2017.11.37

View this article at: http://dx.doi.org/10.21037/jtd.2017.11.37

The European Respiratory Society's Annual Congress 2017, once again, provided an excellent platform to present and discuss hot research topics and challenges in airways diseases. A number of high quality oral and poster sessions discussed novel mechanisms of disease, biomarkers for disease severity, translational and experimental physiology, impact of airway diseases on patients' health status and novel therapeutic options. We discuss the highlights of some key studies in asthma and chronic obstructive pulmonary disease (COPD), which provide novel mechanistic and therapeutic insights.

\section{Asthma update}

Asthma continues to present a number of diagnostic and therapeutic challenges for patients and healthcare providers resulting in a high burden of disease and high costs for the healthcare budget. The mechanisms for the development of early onset childhood, early adult [18-39] and late onset adult asthma $(\geq 40)$ are unclear. Liu et al. (1) presented data from a longitudinal cohort study using the Danish national register of nearly 700,000 participants showed that earlyonset transient, early-onset persistent, and late-onset asthma was more common in offspring of mothers who had asthma compared to fathers. This raises the possibility of postnatal and in utero physiology playing an important role. To characterise the features of adult [18-39] and late onset asthma ( $\geq 40)$, Verhamme et al. (2) analyzed the electronic health records from five European countries to include over 500,000 patients of whom $8 \%$ had severe asthma; $56 \%$ had late onset asthma which rose to $70 \%$ in those with severe asthma. The late onset asthmatic group had fewer atopic subjects, but suffered more frequently with nasal disease, gastro-oesophageal reflux disease (GORD), and were more obese. In another Danish cohort study, 55,000 patients between the ages of 50-64 were followed up to determine the characteristics of asthma-COPD overlap syndrome defined by at least one admission for both asthma and COPD. Information on life style, BMI, education, hormone therapy (HT), occupation and co-morbidities was collected by self-administered questionnaires at baseline. Age, smoking, HT, unemployment, being divorced was associated with increased risk for ACOS whilst physical activity and higher levels of education was associated with lower risk. Although there could be a number of confounders, a broader holistic approach to treatment needs to target lifestyle changes, education and socio-economic factors.

There has been an increasing interest in the role of inflammatory cytokines secreted from the airway epithelium in orchestrating and activating type 2 inflammation. These are collectively known as the epithelial 'alarmins' and currently include IL-33, IL-25 and thymic stromal lymphopoietin (TSLP). Recent evidence has shown that exposure to aero-allergen challenge increases type 2 (T2) inflammation in patients with asthma via activation of TSLP which can be significantly reduced by blocking TSLP (3). Viruses are the commonest causes of asthma exacerbation but the role of viruses on these epithelial alarmins is unclear. Upham et al. (4) presented data to show that exposure of peripheral blood mononuclear cells to rhinovirus 16 and 
IL-33 induced an upregulation of one of the chains of the IL-33 receptor called ST2 on type 2 innate lymphoid cells (ILC2s) and conventional T-cells, which did not occur in samples from healthy donors. This is an important mechanistic finding as ILC2 cells are potent activators of local in situ eosinophilopoesis via IL-5 production, and hence, could help explain how viruses could activate T2 inflammation. In a Canadian study, Sehmi et al. (5) reported that benralizumab (30 $\mathrm{mg}$ s/c for 28 weeks) caused a significant reduction in blood IL5R $\alpha+\mathrm{ILC} 2$ numbers along with reductions in blood and sputum eosinophilia.

There was a focus on the development and use of novel immune therapy targeting T2 inflammation in patients with severe asthma. This group accounts for approximately $8 \%$ of all asthma patients, characterised by recurrent exacerbations and persistent symptoms despite being on high dose inhaled steroid and oral maintenance steroid therapy. A number of centres now use mepolizumab and reslizumab for such patients based on previous data showing improvements in exacerbation frequency, lung function and eosinophil counts. However, data was also presented at the ERS congress to show that compared to placebo, mepolizumab (100 mg) every 4 weeks for 24 weeks showed significant improvements in Global Rating of Activity Limitation and Global Impression of Change. In patients with asthma and allergic rhinitis, dupilumab, an anti-IL4R antibody which inhibits IL-4 and IL-13 signalling, showed a reduction in severe exacerbations, improvement in lung function, and improvements in each of the domains of ACQ-5 score in this sub-group. As chronic steroid use is associated with increasing morbidity, the possibility of a non-steroidal selective glucocorticoid receptor modulator (SGRM) was evaluated in a randomised, placebo controlled, cross-over study. Compared to placebo, inhaled therapy with a SGRM improved lung function by nearly $150 \mathrm{~mL}$, with improvements in all secondary endpoints; FeNO, ACQ-5, peak flow, and rescue salbutamol use. Further long term exacerbation data and evaluation in a broader asthma population is still needed.

\section{COPD update}

Several exciting studies presented data at the ERS Congress on COPD mechanisms, severity assessment, phenotypes and treatment, highlighting the complex and heterogeneous nature of COPD, which is still poorly understood, and the need for development of personalised treatment strategies.
The assessment of COPD severity in daily clinical practice has significantly changed over the last decade. Firstly, the traditional spirometric assessment was supplemented by the evaluation of symptoms burden and exacerbations frequency (GOLD 2013), as the prognostic value of these patient important outcomes was confirmed by large studies such as the ECLIPSE cohort (6). More recently, GOLD 2017 still recognises the prognostic value of spirometry, but downgrades its importance in the direction of pharmacological treatment (7). At the congress, several groups evaluated the differences of subsequent versions of the GOLD severity classification and their impact on patients' management. Firstly, Chalmers and colleagues, in a large record-linkage study of $n=4,885$ patients with COPD who were followed closely for a median of 7 years, from the Scottish regional COPD network, confirmed the prognostic value of GOLD 2013 classification; patients with more severe COPD are at significantly higher risk of death. Interestingly, the mortality of patients with declining COPD severity as per GOLD 2013, decreases. This suggests that improvement of GOLD stage could be used as a treatment aim or outcome measure in future clinical trials. On the other hand, GOLD 2017 demonstrated lower survival discrimination compared to GOLD 2013, in a post-hoc analysis from COPD Gene Cohort [Labaki et al. (8), $\mathrm{n}=4,469$, follow-up $>6$ years]. In addition, reclassification of the same population using GOLD 2017 led to a shift of $12.5 \%$ of the participants from Groups C and $\mathrm{D}$ to Groups $\mathrm{A}$ and $\mathrm{B}$, respectively, recommending less pharmacological treatments for them. This effect was more pronounced in a post-hoc analysis from TONADO and OTEMTO trials [Buhl et al. (9), $\mathrm{n}=5,162$ ] which found a $35 \%$ shift of participants from Groups C/D to Groups A/B, with the application of GOLD 2017 classification (compared to GOLD 2013). Unfortunately, none of these studies presented the outcomes of the groups of participants who were re-classified into milder COPD groups; however, the fact that GOLD 2017 is characterised by a lower survival discrimination, suggests that the mortality of these patients is high, consistent with the mortalities of groups $\mathrm{C}$ and $\mathrm{D}$ and the effects of deescalating their pharmacotherapy (per GOLD 2017), should be carefully evaluated. Finally, Sicilia Torres et al. (10) investigated the prognostic value of exacerbations in $n=819$ patients with COPD who were followed up for a median of 52 months and found that while hospitalized exacerbations are strong predictors of mortality, those treated in the 
community are not associated with mortality and suggested that this should be further assessed and considered in future studies and guidelines.

While current guidelines still adopt an Oslerian approach towards its management, COPD is a complex and heterogeneous disease and the need for the introduction of precision medicine cannot be overstated $(11,12)$. Unfortunately, the significant advances in our understanding of COPD pathophysiology and immunology have barely started to affect clinical practice. Among numerous promising biomarkers that were discussed in the congress, blood eosinophil count (EOS) attracted the most attention. Firstly, Bafadhel et al. (13) in a post-hoc analysis of three randomised controlled trials comparing budesonide + formoterol, formoterol or placebo $(n=4,880)$, modelled the relationship between EOS, annual exacerbation rate, quality of life and $\mathrm{FEV}_{1}$ with budesonide, and found that only COPD patients with EOS $>0.2 \times 10^{9}$ cells $/ \mathrm{L}$ appear to respond to the administration of inhaled corticosteroids (ICS). Whether EOS can predict responsiveness to ICS in COPD has been assessed in post-hoc analyses of several trials, including WISDOM, FLAME, ISOLDE, INSPIRE and TRISTAN, with contradictory results. However, given the significant side effects of ICS, which include a high risk of pneumonia, diabetes and osteoporosis, the strong signal suggesting that EOS might be able to predict responsiveness to ICS warrants thorough evaluation by means of an appropriately powered clinical trial.

Clearly eosinophilic inflammation represents a strong, treatable trait in COPD (11), which needs to be further investigated. Several studies assessing the radiological characteristics of COPD patients with high versus low EOS count were presented at the Congress. Firstly, Papaioannou and colleagues, in a cross-sectional study of 91 well-characterized patients with COPD who underwent lung function and high resolution computed tomography (HRCT) of the chest, found that patients with significant emphysema in HRCT had lower EOS counts, suggesting that different immunological pathways predominate in the development of emphysema versus chronic bronchitis. These findings were not supported by Luijk et al. (14), who assessed chest CT scans of 109 patients with end-stage COPD who did not find a significant association between EOS count and the presence of radiologic emphysema. However, Luijk compared patients with high versus low EOS ( $>2 \%$ versus $<2 \%$, categorical variable), whilst Papaioannou used continuous values, which is a more sensitive method. In addition, Luijk demonstrated a strong correlation between EOS count and airway thickness, only among patients lacking significant CT measured emphysema. Further characterization of the high-EOS COPD phenotype is important.

\section{Unmet needs}

Both asthma and COPD, being frequent, burdensome and still sub-optimally managed diseases, are the focus of intensive research. Our understanding of their complex pathophysiology and immunology is advancing fast. Numerous well-conducted studies were presented on these topics in the congress and attracted exciting scientific discussion. The current challenge is to translate the knowledge of the basic science into late phase translational research and prospective precision medicine trials, which are costly and technically more challenging. While numerous promising biomarkers are identified and validated in early studies, our limited experience on biomarker trials significantly delays their introduction in clinical practice (15). Therefore, there is an urgent need to develop relevant skills and capacity, which will allow us to use more rapidly and effectively our fast-advancing basic knowledge.

\section{Acknowledgements}

None.

\section{Footnote}

Conflicts of Interest: The authors have no conflicts of interest to declare.

\section{References}

1. Liu X, Schlünssen V. Parental asthma and the risk of earlyonset transient, early-onset persistent, and late-onset asthma in the offspring. Eur Respir J 2017;50:OA315.

2. Verhamme KM, Engelkes M, de Ridder M, et al. Characteristics of adult onset vs. late onset asthma a multinational database cohort study. Eur Respir J 2017;50:OA316.

3. Gauvreau GM, O'Byrne PM, Boulet LP, et al. Effects of an anti-TSLP antibody on allergen-induced asthmatic responses. N Engl J Med 2014;370:2102-10.

4. Upham J, Jurak L, Xi Y. IL-33 augments rhinovirus- 
induced type 2 immune responses in asthma via selective upregulation of one chain of the IL-33 receptor. Eur Respir J 2017;50:PA567.

5. Sehmi R, Nair P, Mukherjee M, et al. Benralizumab attenuates airway eosinophilopoietic processes in prednisone-dependent asthma. Eur Respir J 2017;50:OA3217.

6. Hurst JR, Vestbo J, Anzueto A, et al. Susceptibility to exacerbation in chronic obstructive pulmonary disease. $\mathrm{N}$ Engl J Med 2010;363:1128-38.

7. Vogelmeier CF, Criner GJ, Martinez FJ, et al. Global Strategy for the Diagnosis, Management, and Prevention of Chronic Obstructive Lung Disease 2017 Report: GOLD Executive Summary. Eur Respir J 2017;49. pii: 1700214.

8. Labaki W, Martinez C, Regan E, et al. Mortality and exacerbation prediction by GOLD groups ABCD 2011 vs. 2017: analysis of the COPDGene cohort. Eur Respir J 2017;50:OA1494.

9. Buhl R, Maltais F, Abrahams R, et al. Impact of grouping patients by 2017 GOLD strategy assessment criteria: a post hoc analysis of the TONADO and OTEMTO trials.
Eur Respir J 2017;50:OA1497.

10. Sicilia Torres R, Cabrera C, Marín JM, et al. Changes in GOLD Classifications: 2011 versus 2017. Do exacerbations have any role in mortality? Eur Respir J 2017;50:OA1498.

11. Agusti A, Bel E, Thomas M, et al. Treatable traits: toward precision medicine of chronic airway diseases. Eur Respir J 2016;47:410-9.

12. Mathioudakis AG, Chatzimavridou-Grigoriadou V, Corlateanu A, et al. Procalcitonin to guide antibiotic administration in COPD exacerbations: a meta-analysis. Eur Respir Rev 2017;26. pii: 160073.

13. Bafadhel M, Peterson S, De Blas M, et al. Modelling peripheral blood eosinophils to identify response to budesonide in COPD: a post-hoc analysis. Eur Respir J 2017;50:PA394.

14. Luijk HD, Pompe E, Jansen J, et al. Analysis of blood eosinophil levels in relation to CT measured airway wall thickness and emphysema in end-stage COPD. Eur Respir J 2017;50:PA792.

15. Martz L. The FDA's push for better biomarkers. SciBX 2014;7:1060.
Cite this article as: Mathioudakis AG, Satia I, Adcock IM. Airways diseases: insights from the European Respiratory Society Annual Congress 2017. J Thorac Dis 2017;9(Suppl 16):S1550-S1553. doi: 10.21037/jtd.2017.11.37 